\title{
Epigenetic alterations of the $I g f 2$ promoter and the effect of miR-483-5p on its target gene expression in esophageal squamous cell carcinoma
}

\author{
HAN ZHANG $^{1 *}$, XIAOWEI SHI $^{1 *}$, WEIDONG CHANG ${ }^{2},{\text { YINGYING } \text { LI }^{2}, \text { LI WANG }^{2} \text { and LINSONG WANG }}^{1,2}$ \\ ${ }^{1}$ School of Life Sciences and Biotechnology, Sanquan College of Xinxiang Medical University; \\ ${ }^{2}$ College of Life Science, Henan Normal University, Xinxiang, Henan 453007, P.R. China
}

Received July 6, 2017; Accepted October 5, 2017

DOI: $10.3892 / \mathrm{mmr} .2017 .8134$

\begin{abstract}
Esophageal squamous cell carcinoma (ESCC) is one of the most widespread malignancies in China. MicroRNAs (miRNAs/miRs) are endogenous evolutionarily-conserved small non-coding RNAs that are able to regulate ESCC formation and deterioration by negatively regulating specific target genes. In the present study, the expression levels of miR-483-5p and its associated mRNAs were measured by quantitative polymerase chain reaction (PCR) analysis, and the methylation levels of the insulin-like growth factor 2 (Igf2) promoter were detected via the methylation-specific PCR method in serum and tissues from patients with ESCC. The results demonstrated that the expression level of miR-483-5p was significantly upregulated in preoperative serum and cancer tissues from patients with ESCC $(\mathrm{P}<0.01)$, and the miR-483-5p expression levels were correlated with the tumor, node, metastasis stage $(\mathrm{P}<0.05)$ and lymph node metastasis $(\mathrm{P}<0.05)$. In addition, the mRNA levels of miR-483-5p target genes (Rho GDP dissociation inhibitor $\alpha$, activated leukocyte cell adhesion molecule, and suppressor of cytokine signaling 3 ) in cancer tissues were significantly decreased compared with adjacent non-cancerous tissues. These results indicated that miR-483-5p and its target genes may be involved in the developmental process of ESCC. The Igf 2 levels in cancer tissues were significantly increased compared with adjacent non-cancerous tissues $(\mathrm{P}<0.01)$. Additionally, the methylation levels of the Igf2 promoter region were 31.82 and $54.55 \%$ in cancer tissues and adjacent non-cancerous tissues, respectively, suggesting that low methylation of the Igf2 gene promoter region may promote
\end{abstract}

Correspondence to: Professor Linsong Wang, College of Life Science, Henan Normal University, 46 East Construction Road, Xinxiang, Henan 453007, P.R. China

E-mail:wls@htu.cn

*Contributed equally

Key words: insulin-like growth factor II, microRNA-483-5p, esophageal squamous cell carcinoma, methylation, gene expression the expression of Igf 2 and miR-483-5p; this, in turn, induces the degradation of miR-483-5p target genes, and leads to the upregulation of oncogenes and the downregulation of tumor suppressors, which promotes the development of ESCC.

\section{Introduction}

Esophageal cancer refers to upper gastrointestinal tract tumors of epithelial cellular origin. It is the 6th most common cause of cancer-associated mortality, and the eighth most common malignancy worldwide $(1,2)$. In China, it is the 5 th most common cancer and the 4th most common cause of cancer-associated mortality $(3,4)$. Early detection of the disease is essential to improve the survival of patients with esophageal cancer.

MicroRNAs (miRNAs/miRs) are endogenous, single-stran ded non-coding RNAs with 19-25 nucleotides, acting as important mediators in the regulation of gene expression, cell differentiation, the cell cycle and apoptosis $(5,6)$. miRNA microarray profiling of human tumors has demonstrated that certain groups of miRNAs may offer opportunities in the identification of novel biomarkers and therapeutic targets $(7,8)$. A number of miRNAs, including miR-21, miR-373 and miR-483, have been reported to be potential biomarkers of esophageal cancer. miR-483 is an intronic miRNA located within the insulin like growth factor 2 (Igf2) gene locus in mammalian cells (9), and has been associated with a diverse set of human pathologies, including cancer (10-12). A polymorphism at the miR-483-5p binding site in the 3'-untranslated region of the basigin gene has been demonstrated to be associated with increased susceptibility to esophageal cancer in a Chinese population (13). However, to the best of the authors' knowledge, there has been no study regarding the mechanism underlying the regulatory role of miR-483-5p in the development of esophageal cancer.

Epigenetic alterations have been a subject of research, due to their involvement in malignant transformation and tumor progression. There has been an increase in basic and applied research into the field of the epigenetic regulation of esophageal cancer development (14-16). The aim of the present study was to clarify the association between miR-483-5p expression in serum and tissues from patient with esophageal cancer, with epigenetic alterations in the Igf 2 promoter, in addition to the effect of imiR-483-5p on target gene expression. 


\section{Materials and methods}

Patients. The study protocol was approved by the Ethics Committee of the Xinxiang Central Hospital (Xinxiang, China). A total of 23 patients (the age range, 25-60 years; median age, 46; 17 males and 6 females.) with esophageal squamous cell carcinoma (ESCC) from the Xinxiang Central Hospital and 50 healthy subjects were recruited to the present study between January 2014 and February 2015. All participants were genetically unrelated ethnic Han Chinese from the same geographic region (Henan, China). The diagnosis of ESCC was confirmed by histopathology in all patients. Written informed consent was obtained from all participants prior to the study. ESCC tissues and adjacent non-cancerous esophageal tissues (at least $5 \mathrm{~cm}$ away from the tumor) from all 23 patients were collected. A total of $3 \mathrm{ml}$ peripheral blood was collected from each participant (including 50 healthy persons and 23 patients with ESCC; patient blood samples were collected prior to surgery and at 7 days post-surgery).

Tissue and serum sample processing and RNA isolation. All tissue samples were collected during surgery, immediately snap-frozen in liquid nitrogen, and stored at $-80^{\circ} \mathrm{C}$ until RNA extraction. Total RNA was isolated using TRIzol ${ }^{\mathrm{TM}}$ (Invitrogen; Thermo Fisher Scientific, Inc., Waltham, MA, USA), according to the manufacturer's instructions.

Peripheral blood was collected in tubes containing separating gel and clot activator, placed in a water bath for $20 \mathrm{~min}$ at $37^{\circ} \mathrm{C}$, and centrifuged at $3,500 \mathrm{x}$ g for $10 \mathrm{~min}$ at room temperature. The supernatants were transferred to Eppendorf tubes. A second centrifugation at $12,000 \mathrm{xg}$ for $10 \mathrm{~min}$ at $4^{\circ} \mathrm{C}$ was performed to completely remove all cellular components. The serum was subsequently aliquoted and stored at $-80^{\circ} \mathrm{C}$ until RNA extraction. All blood samples were processed within $3 \mathrm{~h}$ following collection. Total serum RNA was isolated from $100 \mu 1$ serum and eluted in $300 \mu 1$ RNase-free water using TRIzol (Invitrogen; Thermo Fisher Scientific, Inc.), according to the manufacturer's instructions.

Reverse transcription-quantitative polymerase chain reaction (RT-qPCR) analysis. qPCR for individual miRNAs was performed on independent sets of serum or tissue using a two-step procedure. qPCR for miRNA Stem-Loop ${ }^{\mathrm{TM}} \mathrm{RT}$ primers for miR-483-5p and miR-16-5p were synthesized by Applied Biosystems (Thermo Fisher Scientific, Inc.) (Table I). A PrimeScript ${ }^{\mathrm{TM}}$ RT reagent kit (Perfect Real Time; Takara Biotechnology Co., Ltd., Dalian, China) was used to reverse transcribe the total RNA. A SYBR Green (Takara Biotechnology Co., Ltd.) qPCR assay kit was used to detect the expression of miR-483-5p and miR-16-5p. The qPCR reaction was performed over 45 cycles $\left(95^{\circ} \mathrm{C}, 10 \mathrm{sec} ; 60^{\circ} \mathrm{C}\right.$, $30 \mathrm{sec}$ ) following an initial denaturation step $\left(95^{\circ} \mathrm{C}, 5 \mathrm{~min}\right)$ on the CFX96 system using Bio-Rad CFX Manager 2.0 Software (Bio-Rad Laboratories, Inc., Hercules, CA, USA). The expression levels of miRNA were calculated and quantified using the $2^{-\Delta \Delta \mathrm{Cq}}$ method (17). miR-16-5p was used as the internal control. All reactions were performed in triplicate.

Prediction of miR-483-5p target genes. miR-483-5p target genes were predicted using miRBase (www.mirbase.org),
Table I. Primer sequences.

Primer

Sequence

miR-483-5p-RT

GTCGTATCCATGGCAGGGTCCGAG
GTATTCGCCATGGATACGACCTCCCT

miR-483-5p-F

GCAAGACGGGAGGAAAGAAGGGA

universal reverse TGGCAGGGTCCGAGGT

GAPDH-F

GAPDH-R

GCACCGTCAAGGCTGAGAAC

Socs3-F

TGGTGAAGACGCCAGTGGA

Socs3-R

ALCAM-F

ALCAM-R

ARHGDIA-F

CAGGAATGTAGCAGCGATGGAA

ARHGDIA-R

CCTGTCCAGCCCAATACCTGA

Igf2-F

Igf2-R

miR-16-RT

CCTTGTTGCTGGTGTCGTCTACT ATTACCGAGGTCCTTGTTTACATGT

miR-16-F

Igf2-MF3

Igf2-MR3

Igf2-UF3

AACCGAGAGATAGTGTCCGGC

TCTTGACGCCTTTCCTGTACG

CCGTGCTTCCGGACAACTT

CTGCTTCCAGGTGTCATATTGG

GTCGTATCCATGGCAGGGTCCGAGGT ATTCGCCATGGATACGACCGCCAAT GCGGTAGCAGCACGTAAATATT

Igf2-UR3

AGCGGTTTCGGTGTCGTTATC CGAACGCCCAACTCGATT

GGATTGTGGGTGTTTAGTTTGGTT

CCTTTCCACACTACATCCCAAAA

F, forward; R, reverse; RT, reverse transcription, ALCAM, activated leukocyte cell adhesion molecule; ARHGDIA, Rho GDP dissociation inhibitor $\alpha$; Socs 3, suppressor of cytokine signaling 3; Igf2, insulin-like growth factor 2; miR, microRNA; M, methylated; $\mathrm{U}$, unmethylated.

Target Scan (www.targetscan.org), and PicTar (pictar. mdc-berlin.de).

Genomic DNA isolation and methylation analysis. Genomic DNA was extracted using an EZ DNA Methylation-Gold ${ }^{\mathrm{TM}}$ kit (Qiagen GmbH, Hilden, Germany). The methylation status of the Igf2 gene was determined using the methylation-specific PCR (MSP) method on bisulfate-treated genomic DNA. The primers specific for either unmethylated or methylated alleles are listed in Table I. As an internal control, all purified genomic DNA samples were successfully tested by PCR with a Takara EpiTaq ${ }^{\mathrm{TM}}$ HS kit (for bisulfite-treated DNA; Takara Biotechnology Co., Ltd.). Methylated and unmethylated DNAs of normal human peripheral lymphocytes were used as a positive control for the methylated and as a negative control for unmethylated genes, respectively. Samples with $\mathrm{H}_{2} \mathrm{O}_{2}$ instead of DNA were included for each PCR set. PCR products were analyzed on a $1 \%$ agarose gel, stained with ethidium bromide, and visualized under ultraviolet light (DL 2000 Marker; Genstar, Beijing, China). Each MSP was repeated at least once to confirm the results.

Statistical analysis. Data were reported as mean \pm standard deviation for quantitative variables. The difference in mRNA or miRNA expression levels between paired tissue samples was 
A

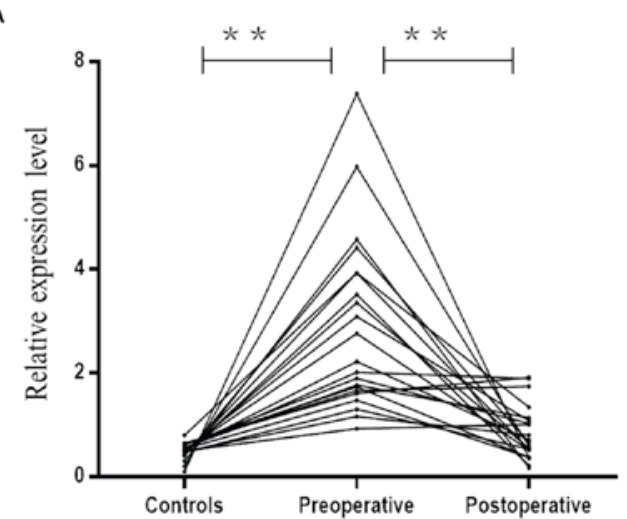

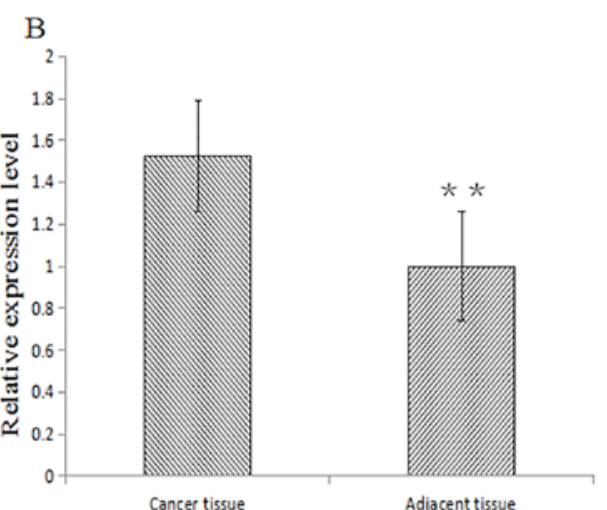

C

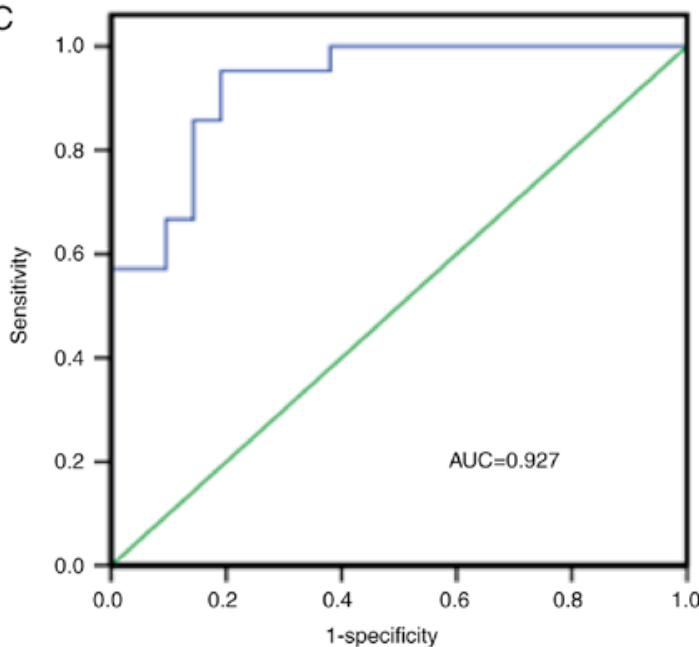

Figure 1. Expression levels of miR-483-5p in ESCC. The data are presented as the mean \pm standard deviation. The asterisks denote a response that was significantly different from the control ("* $\mathrm{P}<0.01)$. (A) The expression level of miR-483-5p in the serum samples from healthy persons and patients with ESCC prior to and post-surgery. (B) The average expression level of miR-483-5p in ESCC tissues and adjacent normal tissues. ${ }^{* *} \mathrm{P}<0.01$ vs. cancer tissue. (C) Receiver operating characteristic curve analysis of serum miR-483-5p. ESCC, esophageal squamous cell carcinoma; miR, microRNA; AUC, area under the curve.

calculated using the Wilcoxon matched-pairs test. Correlations between independent samplings and qPCR analysis of Igf2 and miRNA were determined by the Spearman correlation test. The Mann-Whitney test was performed to determine the significance of serum miRNA levels. The area under the curve (AUC) for tissue and serum miRNAs was determined using receiver operator characteristic (ROC) analysis. $\mathrm{P}<0.05$ was considered to indicate a statistically significant difference. The statistical analysis was performed using SPSS 16.0 software (SPSS, Inc., Chicago, IL, USA).

\section{Results}

Analysis of the expression levels of miR-483-5p in ESCC. To investigate the role of miR-483-5p in ESCC, the miR-483-5p expression levels were evaluated in the serum from patients with ESCC patients prior to and following surgery, and from ESCC-free subjects. The results demonstrated that miR-483-5p was highly expressed in the serum prior to surgery in patients with ESCC, which was significantly increased compared with those following surgery in patients with ESCC and normal subjects $(\mathrm{P}<0.05$; Fig. 1A).

The expression level of miR-483-5p in cancer tissues of patients with ESCC was significantly increased compared with those in adjacent non-cancerous tissues $(\mathrm{P}<0.01)$, and the
Table II. Correlation of miR-483-5p with clinical features in patients with esophageal squamous cell carcinoma.

\begin{tabular}{lccc}
\hline & & \multicolumn{2}{c}{ miR-483-5p } \\
\cline { 3 - 4 } Characteristic & No. cases & $\%$ & P-value \\
\hline TNM stage & & & 0.033 \\
I & 3 & 11.5 & \\
II & 8 & 30.8 & \\
III & 13 & 50.0 & \\
IV & 2 & 7.7 & \\
Lymph node metastasis & & & 0.048 \\
No & 11 & 42.3 & \\
Yes & 15 & 57.7 & \\
\hline
\end{tabular}

TNM, tumor, node, metastasis; miR, microRNA.

difference between cancer tissues was similar to that between the serum samples (Fig. 1B). The expression level of miR-483-5p was positively correlated with the clinical tumor, node, metastasis (TNM) staging of patients with ESCC $(\mathrm{P}<0.05)$, and with the degree of lymph node metastasis $(\mathrm{P}<0.05$; Table II). 


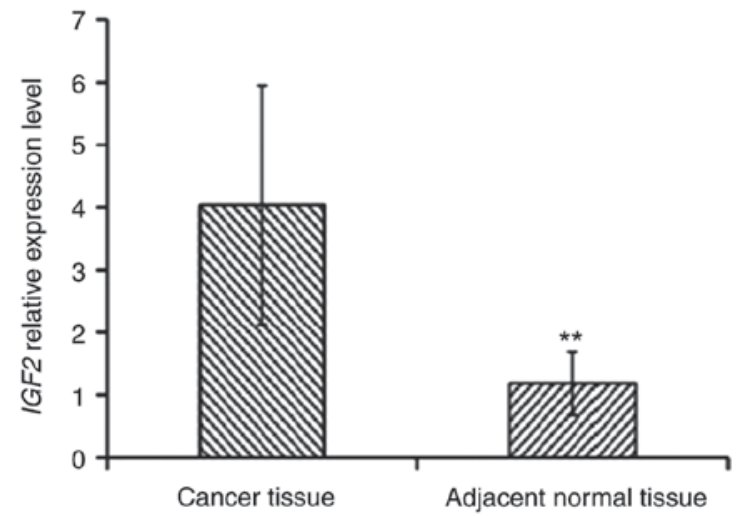

Figure 2. Expression levels of IGF2 in esophageal squamous cell carcinoma. The data are presented as the mean \pm standard deviation. ${ }^{* *} \mathrm{P}<0.01$ vs. cancer tissue. IGF2, insulin-like growth factor 2 .

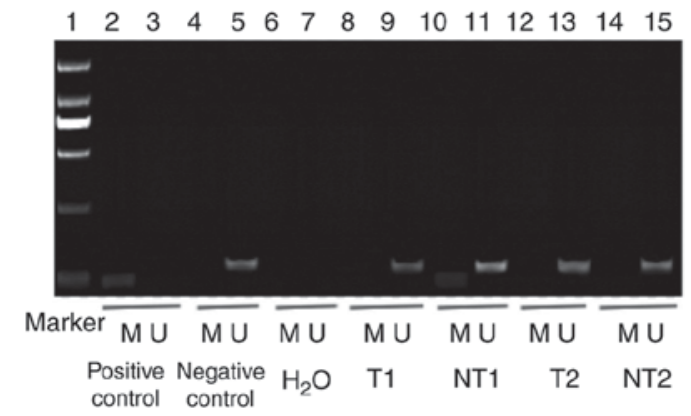

$\mathrm{B}$

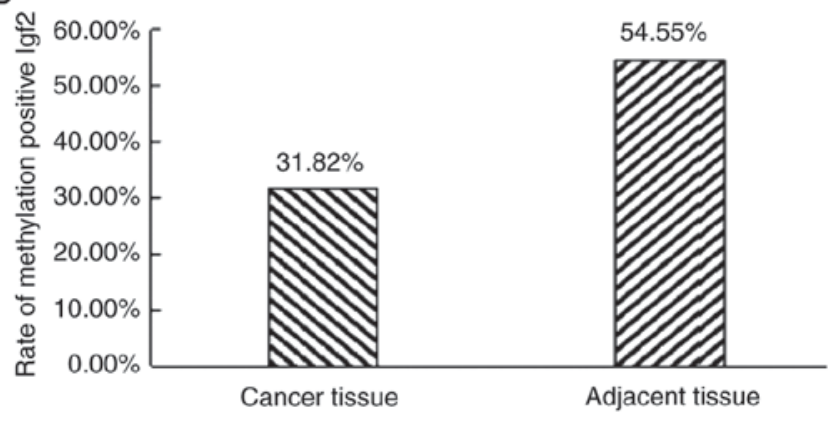

Figure 3. DNA promoter methylation analysis. (A) Detection of methylation status of the IGF2 gene promoter region (Marker DL 2,000). Lane 1, marker (DL 2,000); lane 2, positive control of DNA methylation in peripheral blood lymphocytes from healthy people; lane 3, negative control of DNA methylation in peripheral blood lymphocytes from healthy people; lane 4, positive control of DNA nonmethylation in peripheral blood lymphocytes from healthy people; lane 5, negative control of DNA nonmethylation in peripheral blood lymphocytes from healthy people; lane 6 , distilled water as the negative control of the methylated template; lane 7 , distilled water as a template for the negative control of nonmethylation; lane 8, methylation of cancer tissue; lane 9, nonmethylation of cancer tissue; lane 10, methylation of adjacent normal tissue; lane 11, nonmethylation of adjacent normal tissue; lane 12, methylation of cancer tissue; lane 13, nonmethylation of cancer tissue; lane 14, methylation of adjacent normal tissue; and lane 15, nonmethylation of adjacent normal tissue. (B) Methylation positive rate of the IGF2 gene in cancer tissue and adjacent normal tissue from patients with esophageal squamous cell carcinoma. IGF2, insulin-like growth factor 2; M, methylated; $\mathrm{U}$, unmethylated; T, tumor tissues; NT, normal tissues.

In order to analyze the diagnostic potential of serum miRNA in ESCC, the ROC curve and AUC value were analyzed to further assess the reliability of serum miR-483-5p expression levels examined by the qPCR. ROC curve analysis
Position 2407-2414 of ALCAM 3'UTR
hsa-miR-483-5p

Position 475-497 of ARHGDIA 3'UTR

hsa-miR-483-5p

Position 298-325 of Socs3 3'UTR

hsa-miR-483-5p

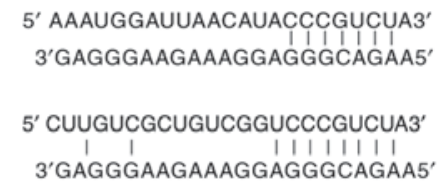

5'CCCCUUCCTCCACACCCCCUCUGCUU3

3'GAGGGAAGAAAGGAGGGCAGAA5'
Figure 4. Prediction of miR-483-5p target genes. Target genes were predicted using miRBase, TargetScan and PicTar. The sequences of putative miR-483-5p binding sites in the 3'-UTRs of ALCAM, ARHGDIA and Socs3 are presented. UTR, untranslated region; ALCAM, activated leukocyte cell adhesion molecule; ARHGDIA, Rho GDP dissociation inhibitor $\alpha$; Socs3, suppressor of cytokine signaling 3; miR, microRNA.

demonstrated that the AUC value of miR-483-5p was 0.927 (95\% confidence interval, 0.85-1.00). When the threshold value was 0.762 , the sensitivity was $95.2 \%$ and the specificity was $81 \%$ (Fig. 1C). AUC may be used as an indicator for the accurate evaluation of certain diagnostic methods, and used for clinical diagnostic tests. A larger AUC value indicates a greater diagnostic value. The closer the AUC is to 1 , the higher its accuracy is, suggesting that serum miR-483-5p may be considered to be a diagnostic marker in ESCC.

Analysis of Igf2 gene expression and promoter region methylation. It has been reported in the literature that miR-483 is located in the second intron of Igf2, and that the expression levels of Igf2 directly affect the expression of miR-483. Therefore, the expression of the Igf 2 gene and the methylation levels in its promoter region were examined. The experimental results demonstrated that the expression levels of Igf2 in cancer tissues of patients with ESCC were significantly increased compared with those in paracancerous tissues $(\mathrm{P}<0.01 ;$ Fig. 2). The methylation level of the Igf 2 promoter region was decreased in tumor tissues $(31.82 \%)$ compared with adjacent non-cancerous tissues (54.55\%; Fig. 3).

Analysis of the expression of miR-483-5p target genes. miRNAs exert their functions primarily by affecting the expression of their target genes. A bioinformatical analysis was performed for the target genes of miR-483-5p (Fig. 4). A total of three miR-483-5p target genes including Rho GDP dissociation inhibitor $\alpha$ (ARHGDIA), activated leukocyte cell adhesion molecule (ALCAM) and suppressor of cytokine signaling 3 (Socs3) were selected for further analysis. The results demonstrated that the expression levels of the three target genes in cancer tissues was significantly decreased compared with adjacent non-cancerous tissues, implying that miR-483-5p may influence the expression of these genes (Fig. 5).

\section{Discussion}

The study of miRNAs has been extended into numerous types of tumor. The expression of miR-483 has been demonstrated to be upregulated in approximately one-half of human tumors (18), including adrenocortical carcinoma and hepatocellular carcinoma $(11,19)$, and its oncogenic targets, including 

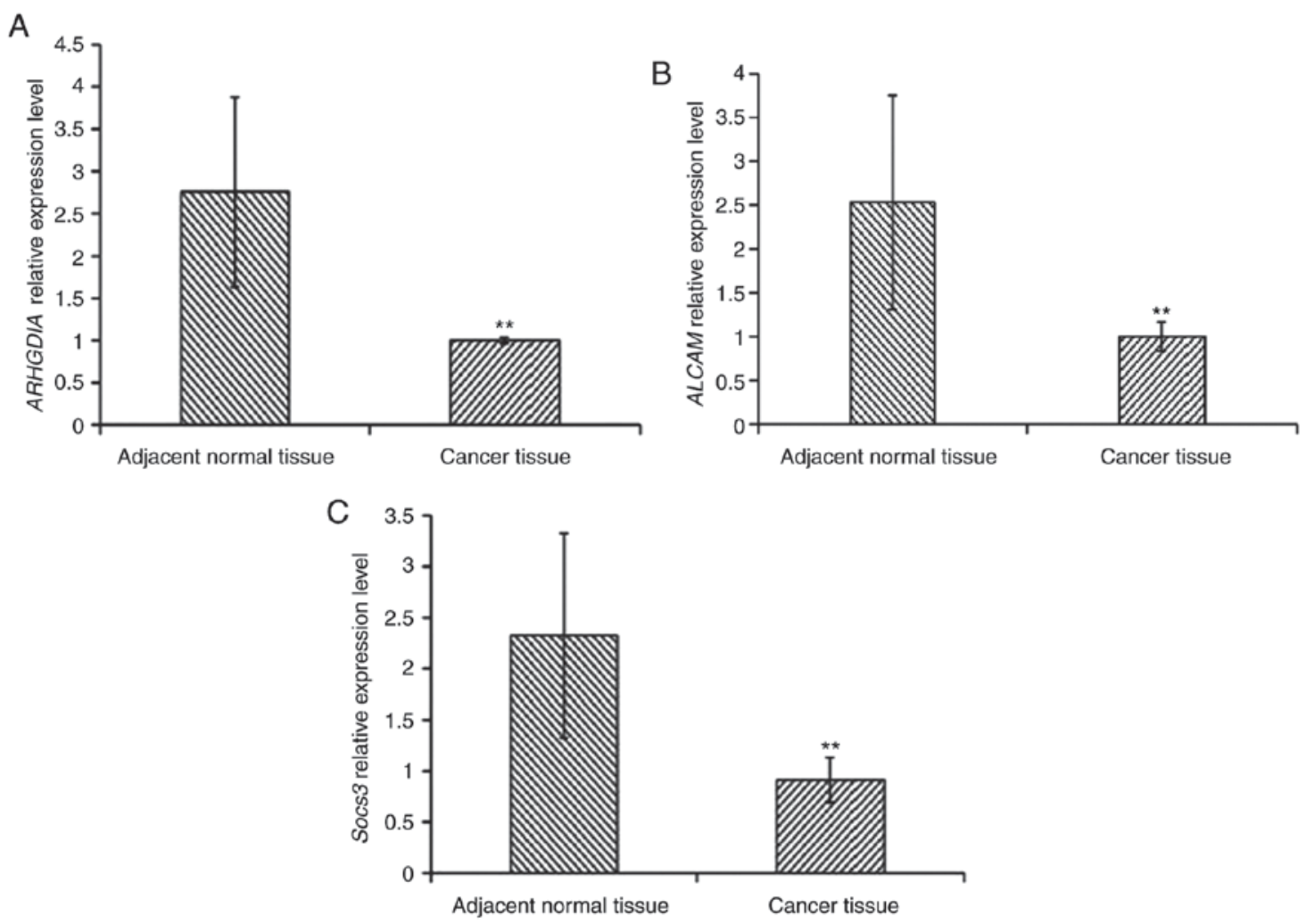

Figure 5. Expression levels of ARHGDIA, ALCAM and Socs3 in ESCC. The data are presented as the mean \pm standard deviation. (A) The expression levels of ARHGDIA in tissues from patients with ESCC. (B) The expression levels of ALCAM in tissues from patients with ESCC. (C) The expression levels of Socs3 in tissues from patients with ESCC. ${ }^{* *} \mathrm{P}<0.01$ vs. adjacent normal tissue. ESCC, esophageal squamous cell carcinoma; ALCAM, activated leukocyte cell adhesion molecule; ARHGDIA, Rho GDP dissociation inhibitor $\alpha$; Socs3, suppressor of cytokine signaling 3.

cellular tumor antigen p53, BCL2 binding component 3 , catenin $\beta 1$, and insulin-like growth factor 1 receptor have been identified (20).

The degree of methylation of the promoter region affects the regional DNA structure and influences gene transcription. The results of the present study demonstrated that the differences in Igf2 promoter methylation resulted in the differential expression of Igf 2 between cancer tissues and paracancerous tissues in patients with ESCC. The methylation level of the Igf 2 promoter region in cancer tissues was low, although Igf2 gene expression was increased. The methylation of the promoter region of Igf 2 in adjacent non-cancerous tissues was high, while the Igf2 expression level was decreased. miR-483-5p is coexpressed with Igf2 (21); thus, the expression of miR-483-5p is enhanced when the expression of Igf 2 is increased. Therefore, the extent of methylation in the host gene promoter region influences miRNA expression, indicating that epigenetic modification serves an important role in the regulation of miRNA expression.

It has been demonstrated that miRNAs are able to bind to their complementary mRNA sites through base-pairing to regulate gene expression (22). Each miRNA has hundreds of evolutionarily conserved or non-conservative target genes. Therefore, appraisal of the miRNA target genes has become a challenge. In the present study, the mRNA levels of miR-483-5p target genes, including ARHGDIA, ALCAM and Socs3, were detected, which demonstrated that the levels of these genes were negatively-associated with the expression of miR-483-5p. However, the expression of these genes was low in cancer tissues, and high in adjacent non-cancerous tissues, suggesting that miR-483-5p may mediate its potential the expression of the target genes ARHGDIA, ALCAM and Socs3, resulting in decreased expression in ESCC.

A recent study demonstrated that ARHGDIA may be a candidate tumor suppressor, and that it was downregulated in hepatoma and mammary cancer (23). Downregulation of ARHGDIA may reverse the activity of Rac family small GTPase 1 and cell division cycle 42, and increase cell migration and invasion to promote tumor metastasis (24). In the present study, the expression levels of miR-483-5p correlated with TNM stage and lymph node metastasis, suggesting that miR-483-5p may promote the development of ESCC by downregulating the target gene ARHGDIA.

ALCAM is involved in homotypic or heterotypic cellular adsorption. The expression levels of ALCAM vary in distinct cancer tissues or at distinct stages of tumor progression (25-27). Olson et al (18) reported a negative correlation between ALCAM levels and the degree of tumor malignancy, and ALCAM expression is elevated in early ESCC (25). Therefore, the reduction of ALCAM expression may be due to the fact that the majority of samples in the present study were advanced ESCC.

The Socs 3 gene belongs to the cytokine signaling inhibitor protein family. The Socs 3 protein is able to negatively regulate the signaling processes of insulin and a number of cytokines to regulate immune reactions, inflammation and lymphocyte differentiation (28). Similarly, miR-483 negatively regulates the target gene Socs 3 to regulate liver cancer cell proliferation 
and development (29). The results of the present study demonstrated that miR-483-5p exhibited high expression, although Socs 3 exhibited low expression, in ESCC cancer tissues, indicating that Socs 3 may serve a role in ESCC pathogenesis.

In conclusion, the results of the present study suggested that miR-483-5p may be involved in ESCC pathogenesis, and that low methylation of the Igf 2 gene promoter region led to increased expression of Igf 2 and miR-483-5p in ESCC. As a result, the decrease in the ARHGDIA, ALCAM and Socs3 expression levels may cause the upregulation of oncogenes and downregulation of tumor suppressors, thereby inducing ESCC. Further studies are required to investigate the detailed mechanism and function of miR-483-5p in ESCC.

\section{Acknowledgements}

The present study was supported by the Key Projects of Science and Technology in Henan Province (grant no. 172102310407) and the Key Research Projects of Henan Higher Education Institutions (grant no. 16A180028).

\section{References}

1. Pennathur A, Gibson MK, Jobe BA and Luketich JD: Oesophageal carcinoma. Lancet 381: 400-412, 2013.

2. Wen SW, Zhang YF, Li Y,Liu ZX, Lv HL, Li ZH, Xu YZ,Zhu YG and Tian ZQ: Characterization and effects of miR-21 expression in esophageal cancer. Genet Mol Res 14: 8810-8818, 2015.

3. Chen W, He Y, Zheng R, Zhang S, Zeng H, Zou X and He J: Esophageal cancer incidence and mortality in China, 2009. J Thorac Dis 5: 19-26, 2013.

4. Peng JZ, Xue L, Liu DG and Lin YH: Association of the p53 Arg72Pro polymorphism with esophageal cancer in Chinese populations: A meta-analysis. Genet Mol Res 14: 9024-9033, 2015.

5. Calin GA and Croce CM: MicroRNA signatures in human cancers. Nat Rev Cancer 6: 857-866, 2006.

6. Garzon R and Croce CM: MicroRNAs and cancer: Introduction. Semin Oncol 38: 721-723, 2011.

7. Mulrane L, Klinger R, McGee SF, Gallagher WM and O'Connor DP: microRNAs: A new class of breast cancer biomarkers. Expert Rev Mol Diagn 14: 347-363, 2014.

8. Ma $\mathrm{J}$ and Li X: MicroRNAs are involved in the toxicity of microcystins. Toxin Rev 36: 165-175, 2017.

9. Fu H, Tie Y, Xu C, Zhang Z, Zhu J, Shi Y, Jiang H, Sun Z and Zheng X: Identification of human fetal liver miRNAs by a novel method. FEBS Lett 579: 3849-3854, 2005.

10. De-Ugarte L, Yoskovitz G, Balcells S, Güerri-Fernández R, Martinez-Diaz S, Mellibovsky L, Urreizti R, Nogués X, Grinberg D, García-Giralt N and Díez-Pérez A: miRNA profiling of whole trabecular bone: Identification of osteoporosis-related changes in miRNAs in human hip bones. BMC Med Genomics 8 : $75,2015$.

11. Veronese A, Lupini L, Consiglio J, Visone R, Ferracin M, Fornari F, Zanesi N, Alder H, D'Elia G, Gramantieri L, et al: Oncogenic role of miR-483-3p at the IGF2/483 locus. Cancer Res 70: 3140-3149, 2010.

12. Wang $\mathrm{C}$, Sun Y, Wu H, Zhao D and Chen J: Distinguishing adrenal corticalcarcinomas and adenomas: A study of clinicopathological features and biomarkers. Histopathology 64: 567-576, 2014.

13. Li HY, Liu YC, Bai YH, Sun M, Wang L, Zhang XB and Cai B: SNP at miR-483-5p-binding site in the 3'-untranslated region of the BSG gene is associated with susceptibility to esophageal cancer in a Chinese population. Genet Mol Res 15: 1-10, 2016.
14. Baba $\mathrm{Y}$, Watanabe $\mathrm{M}$ and $\mathrm{Baba} \mathrm{H}$ : A review of the alterations in DNA methylation in esophageal squamous cell carcinoma. Surgery today 43: 1355-1364, 2013.

15. Toh Y, NEgashira A and Yamamoto M: Epigenetic alterations and their clinical implications in esophageal squamous cell carcinoma. Gen Thorac Cardiovasc Surg 61: 262-269, 2013.

16. Wang K, Johnson A, Ali SM, Klempner SJ, Bekaii-Saab T, Vacirca JL, Khaira D, Yelensky R, Chmielecki J, Elvin JA, et al: Comprehensive genomic profiling of advanced esophageal squamous cell carcinomas and esophageal adenocarcinomas reveals similarities and differences. Oncologist 20: 1132-1139, 2015.

17. Livak KJ and Schmittgen TD: Analysis of relative gene expression data using real-time quantitative PCR and the 2(-Delta Delta C(T)) method. Methods 25: 402-408, 2001.

18. Olson P, Lu J, Zhang H, Shai A, Chun MG, Wang Y, Libutti SK, Nakakura EK, Golub TR and Hanahan D: MicroRNA dynamics in the stages of tumorigenesis correlate with hallmark capabilities of cancer. Genes Dev 23: 2152-2165, 2009.

19. Soon PS, Tacon LJ, Gill AJ, Bambach CP, Sywak MS, Campbell PR, Yeh MW, Wong SG, Clifton-Bligh RJ, Robinson BG and Sidhu SB: miR-195 and miR-483-5p identified as predictors of poor prognosis in adrenocortical cancer. Clin Cancer Res 15: 7684-7692, 2009.

20. Li F, Ma N, Zhao R, Wu G, Zhang Y, Qiao Y, Han D, Xu Y, Xiang Y, Yan B, et al: Overexpression of miR-483-5p/3p cooperate to inhibit mouse liver fibrosis by suppressing the TGF- $\beta$ stimulated HSCs in transgenic mice. Cell Mol Med 18: 966-974, 2014.

21. Ma N, Wang X, Qiao Y, Li F, Hui Y, Zou C, Jin J, Lv G, Peng Y, Wang L, et al: Coexpression of an intronic microRNA and its host gene reveals a potential role for miR-483-5p as an IGF2 partner. Mol Cell Endocrinol 333: 96-101, 2011.

22. Srinivasan S, Selvan ST, Archunan G, Gulyas B and Padmanabhan P: MicroRNAs-the next generation therapeutic targets in human diseases. Theranostics 3: 930-942, 2013.

23. Song Q, Xu Y, Yang C, Chen Z, Jia C, Chen J, Zhang Y, Lai P, Fan $\mathrm{X}$, Zhou $\mathrm{X}$, et al: miR-483-5p promotes invasion and metastasis of lung adenocarcinoma by targeting RhoGDI1 and ALCAM. Cancer Res 74: 3031-3042, 2014.

24. Tovar V, Alsinet C, Villanueva A, Hoshida Y, Chiang DY, Solé M, Thung S, Moyano S, Toffanin S, Mínguez B, et al: IGF activation in a molecular subclass of hepatocellular carcinoma and pre-clinical efficacy of IGF-1R blockage. Hepatol 52: 550-559, 2010.

25. Verma A, Shukla NK, Deo SV, Gupta SD and Ralhan R: MEMD/ALCAM: A potential marker for tumor invasion and nodal metastasis in esophageal squamous cell carcinoma. Oncology 68: 462-470, 2005.

26. EskandariL, Akbarzadeh A,Zarghami N and Rahmati-Yamchi M: Gold nanoprobe-based method for sensing activated leukocyte cell adhesion molecule (ALCAM) gene expression, as a breast cancer biomarker. Artif Cells Nanomed Biotechnol 45: 277-282, 2017.

27. Hui B, Chen X, Hui L, Xi R and Zhang X: Serum miRNA expression in patients with esophageal squamous cell carcinoma. Oncol Lett 10: 3008-3012, 2015.

28. Shi L, Liu S, Zhao W and Shi J: miR-483-5p and miR-486-5p are down-regulated in cumulus cells of metaphase II oocytes from women with polycystic ovary syndrome. Reprod Biomed Online 31: 565-572, 2015.

29. Jorgensen SB, O'Neill HM, Sylow L, Honeyman J, Hewitt KA, Palanivel R, Fullerton MD, Öberg L, Balendran A and Galic S: Deletion of skeletal muscle SOCS3 prevents insulin resistance in obesity. Diabetes 62: 56-64, 2013.

This work is licensed under a Creative Commons Attribution-NonCommercial-NoDerivatives 4.0 International (CC BY-NC-ND 4.0) License. 